compared with a threshold of $>10 \mu \mathrm{g} / \mathrm{g}$, with an increased specificity at a threshold of $>10 \mu \mathrm{g} / \mathrm{g}$ (table 1$)$.

Conclusions A lower threshold of FIT $>4 \mu \mathrm{g} / \mathrm{g}$ and anaemia in our population did not improve detection of significant pathology.

\section{PTH-102 BOWEL CANCER SCREENING; VARIATIONS IN ADENOMA DETECTION RATES, CANCER RATES, AND COMFORT SCORES BY ETHNICITY}

\begin{abstract}
${ }^{1}$ Maurice Dungey, ${ }^{2}$ Mina Hanna, ${ }^{3}$ Vicki Lewin, ${ }^{4}$ Nalin Natarajan, ${ }^{5}$ Benjamin Robinson, ${ }^{1}$ Richard Robinson, ${ }^{3}$ Ajay Verma*. 'University Hospitals Leicester NHS Trust, Leicester, UK; ${ }^{2}$ University Hospital Lewisham, London, UK; ${ }^{3}$ Kettering General Hospital NHS Foundation Trust, Kettering, UK; ${ }^{4}$ University Hospital London NHS foundation trust, London, UK; ${ }^{5}$ County Durham and Darlington NHS foundation trust, Durham, UK
\end{abstract}

\subsection{6/gutjnl-2021-BSG.305}

Introduction Colonoscopy is the investigation of choice after a positive stool test for occult blood (faecal occult blood test or faecal immunochemical test) as part of the Bowel Cancer Screening Programme (BCSP). Uptake of bowel cancer screening can be influenced by many factors; one such factor is ethnicity. ${ }^{1}$ In the BCSP, subject's ethnicity is not routinely recorded by the specialist screening practitioners at the clinic appointment prior to having a colonoscopy. Therefore, data on differences in key performance indicators between different ethnic groups is poor.

Aims \& Methods The purpose of this study was to assess differences in adenoma and cancer detection rates and comfort scores within the BCSP.

1508 subjects with a positive faecal occult blood test selfreported their ethnicity prior to colonoscopy. Demographic and endoscopic data was retrieved from the Exeter online database (which is used to record BCSP data). Multivariate logistic regression was used to examine independent variables (age, sex, ethnicity, colonoscopy findings) as predictors of discomfort, adenoma and cancer detection.

The number of non-white/non-South Asian subjects was too small for meaningful comparison, we have analysed differences between white and South Asian populations.

Results The adenoma detection rate (ADR) was greater in White than South Asian patients $(43.5 \%$ vs. 31.9\%; OR: 1.64, $\mathrm{P}=0.015)$; and in Males than Females $(45.7 \%$ vs. 38.0\%; OR: 1.37, $\mathrm{P}=0.003)$.

Similarly, cancer detection was higher in White than South Asian patients (6.9\% vs. 1.7\%; OR: 4.33, $\mathrm{P}=0.042)$. Female patients were more likely to experience significant discomfort

\begin{tabular}{|c|c|c|c|c|c|}
\hline & & $\mathrm{N}$ & $\begin{array}{l}\text { Polyp } \\
\text { detection } \%\end{array}$ & $\begin{array}{l}\text { Adenoma } \\
\text { detection } \%\end{array}$ & $\begin{array}{l}\text { Cancer } \\
\text { detection } \%\end{array}$ \\
\hline Females & Total & 608 & 49.5 & 38.0 & 5.6 \\
\hline Males & Total & 900 & 60.8 & 45.7 & 7.1 \\
\hline White & Females & 545 & 51.8 & 39.3 & 5.9 \\
\hline \multirow[t]{2}{*}{ population } & Males & 819 & 62.3 & 46.3 & 7.6 \\
\hline & Total & 1364 & 57.8 & 43.5 & 6.9 \\
\hline \multirow[t]{3}{*}{ South Asian } & Females & 52 & 25.0 & 23.1 & 1.9 \\
\hline & Males & 67 & 44.8 & 38.8 & 1.5 \\
\hline & Total & 119 & 36.1 & 31.9 & 1.7 \\
\hline \multicolumn{2}{|c|}{ Total population } & 1508 & 56.0 & 42.6 & 6.5 \\
\hline
\end{tabular}

during colonoscopy (OR: 3.08, $\mathrm{P}<0.001$ ), no differences in comfort scores were found between ethnicities.

Conclusion A lower adenoma detection rate and cancer detection rate was found in South Asian patients requiring colonoscopy as part of bowel cancer screening programme compared to White patients. Comfort scores were not dissimilar between ethnicities, although female participants reported greater discomfort than males.

Understanding the differences between the two largest ethnic groups allows funding to be directed towards communication of these findings to South Asian communities, which may help improve poor attendance to screening programmes that is influenced by fear of adverse findings and the procedure itself.

\section{REFERENCE}

1. Palmer CK, Thomas MC, McGregor LM, von Wagner C, Raine R. Understanding low colorectal cancer screening uptake in South Asian faith communities in England-a qualitative study. BMC Public Health 2015;15(1):998-1005.

\section{PTH-103 BIFIDOBACTERIUM LONGUM 35624(R)AND1714(R) IN ADULTS WITH IRRITABLE BOWEL SYNDROME- RELATIONSHIPS TO SYMPTOM SEVERITY AND CO- MORBIDITY}

${ }^{1}$ Eileen Murphy*, ${ }^{1}$ David Groeger, ${ }^{2}$ Ted Dinan, ${ }^{3}$ Eamonn Quigley. ${ }^{1}$ Precisionbiotics Group, Cork, Ireland; ${ }^{2}$ Department of Psychiatry, Cork University Hospital and University College Cork, Cork Ireland, Cork, Ireland; ${ }^{3}$ Lynda K and David M Underwood Center for Digestive Disorders, Division of Gastroenterology and Hepatology, Houston Methodist Hospital and Weill Cornell Medical College, Houston, USA

\subsection{6/gutjnl-2021-BSG.306}

Introduction Anxiety and depression are commonly encountered co-morbidities among IBS sufferers. B. longum $1714^{\circledR}$ strain has been shown to attenuate stress responses in healthy subjects and B. longum $35624^{\circledR}$ has been shown to reduce symptoms in IBS. However, the effect of these two strains in combination on bowel symptoms and psychiatric comorbidities in adults with IBS is unknown.

Method forty female subjects aged 18-55 years with IBS, diagnosed by Rome III criteria, and mild to moderate anxiety and/or depression as determined by the HADs scale were recruited into a prospective, open label study. Subjects received the 'Dual Action' $\left(5 \times 10^{8} \mathrm{cfu} /\right.$ day of each strain) for 8 -weeks, followed by an 8 -week follow up. IBS symptoms were assessed by the IBS-Symptom Severity Scale (IBS-SSS). Improvement in IBS symptoms was defined as a reduction of $>50$ on the IBS-SSS and in anxiety and depression as a change in HADS A or HADS-D score of $\geq 2$.

Results At baseline, the median IBS-SSS score was 250 (IQR:190-315) HADs-Anxiety (HADs-A) score and HADs-

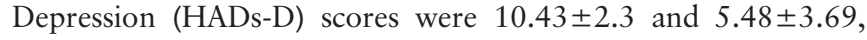
respectively. Correlation analysis of the IBS-SSS to the HADs$\mathrm{D}$ and HADs-A scores showed a weak correlation for HADsD $\left(\mathrm{R}^{2}=0.3246 ; \mathrm{P}=0.001\right)$ but no correlation for HADs-A $\left(\mathrm{R}^{2}=0.3647 ; \mathrm{P}=0.2378\right)$.

At the end of the 8 -week active treatment phase, $85 \%$ of subjects with an IBS-SS score $>175$ had improved irrespective of the type of IBS. There was a significant improvement $(p<0.0001)$ in IBS-SSS as well as the individual Scores of the IBS-SSS such as abdominal pain Severity, abdominal pain frequency, abdominal distension severity, bowel habit satisfaction and quality of life. However once feeding stopped the IBSSSS dis-improved again and by week 16 (8 weeks post 
feeding) with only an improvement of $35 \pm 97$ from baseline. An improvement in HAD score was seen both for depression and Anxiety in 52\% and 55\%, respectively. The HADs-D and HADs-A score improved significantly from baseline to the end of the intervention ( 8 weeks). Surprisingly this improved further once feeding stopped and maintained this improvement by week 16. There was no correlation between HADS-D $\left(\mathrm{R}^{2}=0.001383 ; \quad \mathrm{P}=0.8175\right) \quad$ and HADs- $\mathrm{A} \quad\left(\mathrm{R}^{2}=0.04309 ;\right.$ $\mathrm{P}=0.1928$ ) score and IBS-SSS symptoms at week 8 .

Conclusion Treatment with B. longum $35624^{\circledR}$ and B. longum $1714^{\circledR}$ benefits IBS, regardless of subtype, and also has a beneficial impact on co-morbid anxiety and depression. IBS symptoms and anxiety/depression scores differ in their response time to treatment where an improvement in anxiety/depression was observed in response to the initial improvement in IBS symptoms

\section{PTH-104 COMPARING THE CHARACTERISTICS OF COLORECTAL CANCER IN YOUNG VERSUS OLD PATIENTS}

Farah Jaffar*, Krupa Badiani, Krishan Patel, Jonathan Segal, Rajaratnam Rameshshanker, Arun Rajendran. The Hillingdon Hospital NHS Foundation Trust, London, UK

\subsection{6/gutjnl-2021-BSG.307}

Introduction The overall lifetime risk of developing colorectal cancer (CRC) is approximately $4 \%$ in men and women. Over the past few decades, there has been an increase in incidence of CRC in the young population (defined as 50 years and under) despite an opposite trend in the older population (over 50 years old). Furthermore, more advanced disease has been observed at the point of diagnosis in young patients. Although recognised environmental and genetic factors play a role in this increase, the exact aetiology in this age group is unknown, but is likely multifactorial. Our study aimed to compare the differences in presentations, tumour characteristics and outcomes in patients with CRC according to age group.

Methods A prospectively maintained endoscopy database of all colorectal cancers at a London district general hospital between January 2019 and May 2021 was interrogated in conjunction with our colorectal multidisciplinary team meeting outcomes. Data were collected on age, presentation, indication for initial scope, tumour size and site, and biochemical parameters. We compared differences between the 55 year olds and under with the over 55 year olds. Categorical variables were analysed using the chi-squared test and the Fischer's exact

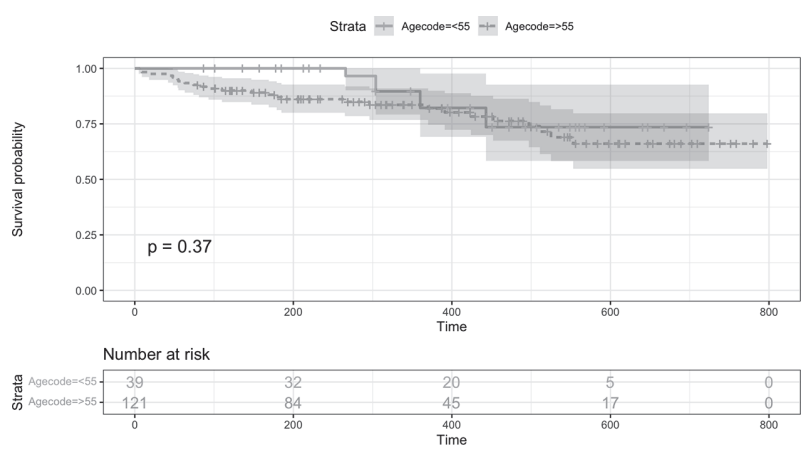

Abstract PTH-104 Figure 1 test. Continuous variable were analysed using two tailed Ttest. Significant values were considered when $\mathrm{p}<0.05$. All data was performed In $\mathrm{R}$ version 1.4.1106.

Results There were 160 patients included in this study. There were 86 females and 74 males. The median age was 72 years old (IQR: 31-94). There were 39 patients in the 55 years and under category and 121 in the over 55 category. There were significant differences in initial presentation with younger patients most commonly presenting with rectal bleeding (33.3\%) and abdominal pain (10.2\%) whereas older patients presented with iron deficiency anaemia (28.1\%) and change in bowel habits (19\%) ( $\mathrm{p}=<0.01)$. Furthermore, there was significantly more lymphovascular invasion in younger patients when compared with older patients $(53.8 \%$ vs $33 \% \mathrm{p}<0.03)$. In the younger population the sigmoid colon was the most common site of malignancy $(25.6 \%)$ compared to the rectum in the older population $(24.0 \%)$. There was no difference in TNM stage at presentation between the two groups $(p=0.93)$. Figure 1 shows the survival analysis between the two groups.

Conclusions Younger patients present differently than older patients with colorectal cancer. Older patients present much more commonly with iron deficiency anaemia compared with younger patients. Furthermore, younger patients present with greater lymphovascular invasion than older patients. Despite differences, survival appears to be similar between the two groups.

\section{PTH-105 SIGMOIDOPEXY-ASSISTED PERCUTANEOUS ENDOSCOPIC COLOSTOMY (PEC) - A STEPWISE IMPROVEMENT IN SAFETY}

John Frost*, Sauid Ishaq, Elizabeth Gibson, Lauren Robinson, Neil Fisher. Russell's Hall Hospital, Dudley, UK

\subsection{6/gutjnl-2021-BSG.308}

Introduction Percutaneous endoscopic colostomy (PEC) is an established alternative to surgery for recurrent sigmoid volvulus or pseudo-obstruction. Tube migration resulting in peritonitis remains a serious safety concern. Endoscopic sigmoidopexy allows fixation of the colon to the abdominal wall prior to PEC insertion, reducing the risk of tube migration or faecal leakage. We present our initial experience of sigmoidopexyassisted PEC.

Methods After endoscopic decompression of the colon, a suitable place for sigmoidopexy is identified (usually mid-sigmoid), using trans-illumination and/or finger indentation.

A triangulated 3-point sigmoidopexy is then performed with a Pexact suturing device. A 15Fr Freka gastrostomy tube is then inserted within the sigmoidopexy points using a standard pull-through technique, to act as the PEC. A flatus bag is attached to the PEC and left on free drainage for 24 hours. Antibiotics are administered peri-procedurally. The sigmoidopexy sutures are removed after 14 days, and the PEC tube is left in situ for a minimum of 3 months.

Results Over an 18 month period 12 patients had sigmoidopexy-assisted PEC (recurrent sigmoid volvulus 9, pseudoobstruction 3) after MDT case selection. All cases were considered unsuitable for surgery after surgical review.

There were no procedural complications and no episodes of faecal peritonitis or other significant sepsis. 